\title{
The Design of Chaos M-ary Communication Demodulator Based on Hamilton Oscillator
}

\author{
${ }^{1} \mathrm{Fu}$ Yongqing and ${ }^{1} \mathrm{Li}$ Xingyuan \\ ${ }^{1}$ College of Information and Communication Engineering, Harbin Engineering \\ University, Harbin 150001, Heilongjiang, China \\ fuyongqing@hrbeu.edu.cn, lixingyuan@hrbeu.edu.cn
}

\begin{abstract}
Previously a novel chaos M-ary digital communication method based on spatiotemporal chaos Hamilton oscillator has been proposed. Without chaos synchronization circumstance, It has performance improvement in bandwidth efficiency, transmission efficiency and anti-white- noise performance compared with traditional communication method. In this paper, the formula for the design of the boundary of zone dividing demodulator in additive white Gaussian noise is derived, besides, the problem about how to determine the boundary of zone dividing demodulator in additive colored noise is deeply studied; Then a solution to construct anti-colored-noise chaos M-ary communication system is proposed, in which a pre-distortion filter is added after the chaos baseband modulator in the transmitter and whitening filter is added before zone dividing demodulator in the receiver. Finally, the chaos M-ary communication system based on Hamilton oscillator is constructed and simulated in different channel noise. The result shows that the proposed method in this paper can improve the anti-colored-noise performance of the whole communication system.
\end{abstract}

Keywords: Hamilton oscillator, zone mapping, zone dividing demodulator, chaos $M$ ary communication, noise

\section{Introduction}

Chaos theory has been developed very rapidly in recent years, and has found an increasingly wide utilization in many fields, such as [1-4]. Chaos is sensitive to initial value, unpredictable and disorderly, these characteristics makes chaotic systems unstable and aperiodic, making them naturally harder to identify and to predict. Recently, many researchers have been looking for ways to utilize the characteristics of chaos in communication systems, since Pecora. [5] Have realized the synchronization between two chaos systems in 1990, chaos communication has become research hotspot.

Although the research of chaos theory on communication application has developed rapidly, the research on $\mathrm{M}$-ary communication scheme are not extensive and thorough enough. In the past four years, some M-ary chaotic spread spectrum communication schemes [6-8] have been proposed. To a certain degree, these schemes have reduced the requirements of the chaos synchronization, and have improved bit error rate performance of the chaotic spread spectrum system, but low bandwidth efficiency is their common shortcoming. Quadrature Differential Chaotic Phase Shift Keying Communication scheme [9] and Orthogonal Chaotic Vector Shift Keying scheme [10] are proposed respectively, these schemes have improved the information transmission rate under the reduced requirements of the chaos synchronization, that is, bandwidth efficiency has been improved to a certain extent, but the bit error rate has not been effectively improved. Literature [11] has proposed the method which separates dynamic chaotic system 
sequences to realize $\mathrm{M}$-ary communication, it improves information transmission rate and bit error rate, but the cost is high system complexity.

In order to solve the above problems, a novel M-ary chaotic digital modulation and demodulation scheme based on Hamilton oscillator has been proposed in our previous research [12]. By using Hamilton map's high sensitivity to initial value and region controllable characteristics of phase trajectory, mapping relationship between digital information and Hamilton map phase trajectory regions is established. Then the digital information is embedded in the phase trajectory regions to complete baseband M-ary digital modulation. Thus the mapping from digital $\mathrm{M}$-ary information to Hamilton oscillator's phase trajectory region is realized. Meanwhile, a digital information demodulation method by judging the phase trajectory region of received signal is proposed, namely zone dividing demodulation (ZDD) method. However, as the working performance of chaos $\mathrm{M}$-ary communication demodulator is so closely connected with channel noise, therefore in order to obtain the optimal design of ZDD, the research on the influences to the roughness of Hamilton modulated signal by the channel noise should be studied deeply. Here the mathematical analysis of designing Hamilton zone dividing demodulator in additive white and colored noise channel is both given. In order to design anti-colored-noise Hamilton chaos M-ary communication, the pre-distortion filter and whitening filter are proposed to construct this system. Finally, the whole chaos 4-ary communication system is constructed on the computer, the result shows that the communication system has better performance in anti-colored-noise and has more secrecy than the original system. Since the proposed ZDD method belongs to non-coherent demodulation technology, no chaos synchronization is needed and symbol error rate performance of the system is improved.

\section{The Construction Method of Chaos M-ary Demodulator in Noise Channel}

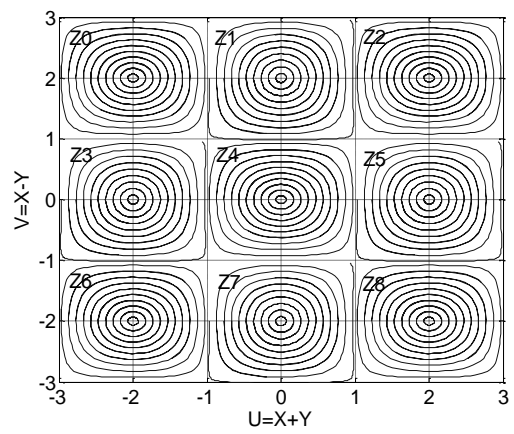

Figure 1. The Part Phase Diagram of Hamilton Oscillator

As shown in Figure 1 [12], Hamilton modulated signals are distributed in the phase trajectories which is in two-dimensional plane, different signals are located into different phase zones, and do not interfere with each other. However, in chaos M-ary communication, when the modulated signals are transmitted to the receiver, the received signals will be disturbed by the channel noise, which can change the shape of the phase trajectory modulated by different digital information. The phase trajectory disturbed by the channel noise may pass through the sub zone, even interfere the other sub zones, which will reduce the demodulating performance of zone dividing demodulator. Here, the boundary problem of demodulator in additive white gauss noise is solved by mathematical analysis. Since the channel noise is close to colored noise in practical engineering application, the solution proposed in literature [12] on how to design zone dividing demodulator can hardly reach the optimal demodulating performance in colored 
noise. Therefore in order to get the optimal demodulating performance of zone dividing demodulator, it is necessary to study the colored noise influence on the phase trajectory of modulated signals.

\subsection{The Study of Zone Dividing Demodulator Boundary in Band-Limited Additive White Noise Channel}

In order to study the colored noise's influence on phase trajectory of chaos signals, firstly the mathematic relationship between band-limited additive white noise and phase trajectory of chaos signals should be constructed.

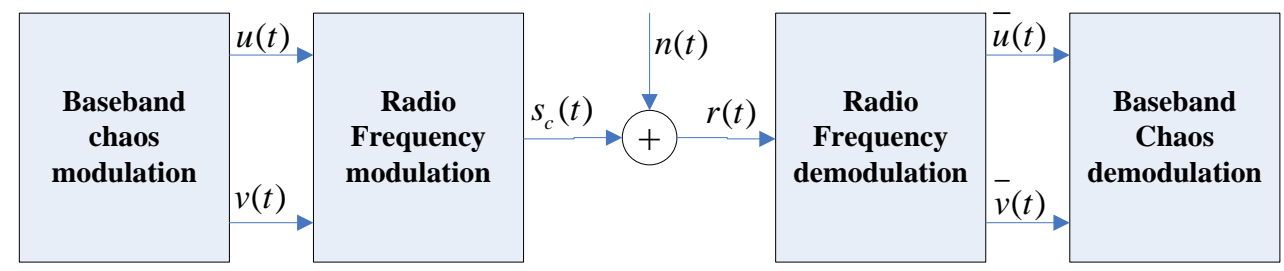

Figure 2. The Structure of Chaos M-Ary Communication

Without loss of generality, the channel is supposed to be Additive White Gaussian Noise (AWGN) channel, with zero mean value and its power spectrum density is $N_{0}$. According to Figure 2, the signal arriving at the receiver can be expressed as:

$$
r(t)=s_{c}(t)+n(t)
$$

where $s_{c}(t)=u(t) \cos \left(\omega_{c} t\right)+v(t) \sin \left(\omega_{c} t\right), \omega_{c}=2 \pi f_{c}$ is carrier angular frequency, $u(t)=J+A \cos \left(2 \pi f_{0} t\right)$ and $v(t)=K+A \sin \left(2 \pi f_{0} t\right)$ denote the Hamilton modulated signals. $n(t)$ will become narrow band Gaussian random process after passing through band-pass filter and it can be expressed as:

$$
\bar{n}(t)=a(t) \cos \left(\omega_{c} t\right)-b(t) \sin \left(\omega_{c} t\right)
$$

where $a(t)$ and $b(t)$ are both stationary random process.

Therefore, after QAM demodulation and lowpass filtering, the baseband signal can be expressed as:

$$
\begin{gathered}
\bar{u}(t)=L P\left\{\left(s_{c}(t)+\bar{n}(t)\right) \cos \left(\omega_{c} t\right)\right\} \\
=\left|\left[(u(t)+a(t)) \cos \left(\omega_{c} t\right)+(v(t)-b(t)) \sin \left(\omega_{c} t\right)\right] \cos \left(\omega_{c} t\right)\right|_{L P F} \\
=\frac{1}{2}[u(t)+a(t)]
\end{gathered}
$$

where $L P\{\square$ represents lowpass filtering, and its cut-off frequency is the maximum of baseband digital information frequency, which is much lower than carrier frequency $\omega_{c}$. In a similar way, we can get:

$$
\bar{v}(t)=\frac{1}{2}[v(t)-b(t)]
$$

Due to that the Hamilton modulated signal recovered by the receiver contains narrow band Gaussian noise, thus the roughness of reconstructed Hamilton phase trajectory will present to be statistic distribution under noise effect.

It is known that the correlation function of narrow band gauss noise appears to be sinc function in time domain, if the correct sample rate is chosen, then the statistical independence between the obtained sample values will be gotten. When the proper time $t$ is given, sampling $\bar{u}(t)$ and $\bar{v}(t)$ we can get: 


$$
\left\{\begin{array}{l}
\bar{u}_{t}=\frac{1}{2}\left(u_{t}+a_{t}\right) \\
\bar{v}_{t}=\frac{1}{2}\left(v_{t}-b_{t}\right)
\end{array}\right.
$$

where $u_{t}$ and $v_{t}$ are deterministic variables, $a_{t}$ and $b_{t}$ are gaussian random variables with zero mean value and $\sigma_{1}^{2}$ variance, thus $\bar{u}_{t}$ and $\bar{v}_{t}$ are also gaussian random variables, their mean value and variance are as follows:

$$
\left\{\begin{array}{c}
E\left[\bar{u}_{t}\right]=\frac{1}{2} E\left[\left(u_{t}+a_{t}\right)\right]=\frac{1}{2} E\left[u_{t}\right]=\frac{1}{2}\left[J+A \cos \left(2 \pi f_{0} t\right)\right] \\
E\left[\bar{v}_{t}\right]=\frac{1}{2} E\left[\left(v_{t}-b_{t}\right)\right]=\frac{1}{2} E\left[v_{t}\right]=\frac{1}{2}\left[K+A \sin \left(2 \pi f_{0} t\right)\right] \\
D\left[\bar{u}_{t}\right]=\frac{1}{4} E\left[\left(a_{t}\right)^{2}\right]=\frac{1}{4} E\left[\left(b_{t}\right)^{2}\right]=D\left[\bar{v}_{t}\right]=\frac{1}{4} \sigma_{1}^{2}
\end{array}\right.
$$

Therefore the probability density functions of $\bar{u}_{t}$ and $\bar{v}_{t}$ can be respectively expressed as:

$$
\left\{\begin{array}{l}
f_{\bar{u}}\left(\bar{u}_{t}\right)=\frac{1}{\sqrt{2 \pi \frac{1}{4} \sigma_{1}^{2}}} \exp \left\{-\frac{\left[\bar{u}_{t}-E\left(\bar{u}_{t}\right)\right]^{2}}{\frac{1}{2} \sigma_{1}^{2}}\right\} \\
f_{\bar{v}}\left(\bar{v}_{t}\right)=\frac{1}{\sqrt{2 \pi \frac{1}{4} \sigma_{1}^{2}}} \exp \left\{-\frac{\left[\bar{v}_{t}-E\left(\bar{v}_{t}\right)\right]^{2}}{\frac{1}{2} \sigma_{1}^{2}}\right\}
\end{array}\right.
$$

Since the mean values of $\bar{u}_{t}$ and $\bar{v}_{t}$ indicate to be the abscissa and ordinate of Hamilton phase trajectory center respectively. Obviously, the position of the center suffers from the effect of channel fading and noise. Let $\bar{u}_{t}-E\left[\bar{u}_{t}\right]$ and $\bar{v}_{t}-E\left[\bar{v}_{t}\right]$ represent the abscissa and ordinate values of Hamilton phase trajectory center under disturbing by the noise, marking $\Delta r_{\bar{u}}=\bar{u}_{t}-E\left[\bar{u}_{t}\right]$ and $\Delta r_{v}=\bar{v}_{t}-E\left[\bar{v}_{t}\right]$. According to probability theory, and in order to demodulate the signal exactly, the degree of confidence is set as follows:

$$
\left\{\begin{array}{l}
P\left\{\frac{\left|\bar{u}_{t}-E\left[\bar{u}_{t}\right]\right|}{\frac{1}{2} \sigma_{1}} \leq \delta_{1}\right\}=0.995 \\
P\left\{\frac{\left|\overline{v_{t}}-E\left[\bar{v}_{t}\right]\right|}{\frac{1}{2} \sigma_{1}} \leq \delta_{2}\right\}=0.995
\end{array}\right.
$$

where equation (9) denotes confidence interval, it means the most possible event's probability that occurs in $\bar{u}_{t}$ and $\bar{v}_{t}$. Since $\bar{u}_{t}$ and $\bar{v}_{t}$ obey gauss distribution with $\left(\frac{1}{2} E\left[\bar{u}_{t}\right], \frac{1}{4} \sigma_{1}^{2}\right)$ and $\left(\frac{1}{2} E\left[\bar{v}_{t}\right], \frac{1}{4} \sigma_{1}^{2}\right)$ respectively, then they obey standard normal distribution after normalization. Thus we can get $\delta_{1}$ and $\delta_{2}$ through looking up standard normal distribution table, where $\delta_{1}=\delta_{2}=2.81$. Therefore we have 


$$
\left\{\begin{array}{l}
\left|\Delta r_{\bar{u}}\right| \leq \frac{1}{2} \delta_{1} \sigma_{1}=1.405 \sigma_{1} \\
\left|\Delta r_{\bar{v}}\right| \leq \frac{1}{2} \delta_{2} \sigma_{1}=1.405 \sigma_{1}
\end{array}\right.
$$

In another hand, the signal-to-noise ratio (SNR) of the whole communication system is expressed as follows:

$$
S N R=\rho=\frac{E_{s a v}}{N_{0}}=\frac{P_{s a v}}{N_{0} B_{w}}=\frac{P_{s a v}}{\sigma_{1}^{2}}
$$

where

$$
P_{\text {sav }}=\frac{1}{T} \int_{0}^{T} s_{c}^{2}(t) d t=\frac{1}{T} \int_{0}^{T}\left[u^{2}(t)+v^{2}(t)\right] \cos ^{2}\left(\omega_{c} t-\theta_{m}\right) d t
$$

$\theta_{m}=\arctan (v / u), B=\frac{1}{T}=R_{m}, T$ is symbol period. Since the signals $u(t)$ and $v(t)$ are given, then $P_{s a v}=\frac{1}{2}\left(J^{2}+K^{2}+A^{2}\right)$, thus

$$
\left\{\begin{array}{l}
\left|\Delta r_{\bar{u}}\right| \leq\left|\Delta r_{\bar{u}}\right|_{\max }=\frac{1}{2} \delta_{1} \sqrt{\frac{P_{s a v}}{\rho}}=1.405 \sqrt{\frac{J^{2}+K^{2}+A^{2}}{2 \rho}} \\
\left|\Delta r_{\bar{v}}\right| \leq\left|\Delta r_{\bar{v}}\right|_{\max }=\frac{1}{2} \delta_{2} \sqrt{\frac{P_{s a v}}{\rho}}=1.405 \sqrt{\frac{J^{2}+K^{2}+A^{2}}{2 \rho}}
\end{array}\right.
$$

The expression gives the relationship between the maximum roughness of the recovered signals' phase trajectory and SNR. Obviously, as SNR increases, $\left|\Delta r_{\bar{u}}\right|_{\max }$ and $\left|\Delta r_{\bar{v}}\right|_{\max }$ will decrease, they have inverse ratio relations.

According to (1) and (12), we can know that the value of $\bar{u}_{t}$ and $\bar{v}_{t}$ have to drop the following range:

$$
\left\{\begin{array}{l}
\frac{1}{2}(J-A)-\left|\Delta r_{\bar{u}}\right|_{\max } \leq \bar{u}(t) \leq \frac{1}{2}(J+A)+\left|\Delta r_{\bar{u}}\right|_{\max } \\
\frac{1}{2}(K-A)-\left|\Delta r_{\bar{v}}\right|_{\max } \leq \bar{v}(t) \leq \frac{1}{2}(K+A)+\left|\Delta r_{\bar{v}}\right|_{\max }
\end{array}\right.
$$

Thus to determine the left-right and up-down boundary range of zone partition demodulator, only $\left|\Delta r_{\bar{u}}\right|_{\max }$ and $\left|\Delta r_{\bar{v}}\right|_{\max }$ are needed. The boundary range of zone partition demodulator can be selected as follows:

$$
\begin{aligned}
& \left\{\begin{array}{l}
u_{L_{0}} \leq \frac{1}{2}(J-A)-\left|\Delta r_{\bar{u}}\right|_{\max } \\
u_{R_{0}} \geq \frac{1}{2}(J+A)+\left|\Delta r_{\bar{u}}\right|_{\max }
\end{array}\right. \\
& \left\{\begin{array}{l}
v_{D_{0}} \leq \frac{1}{2}(K-A)-\left|\Delta r_{\bar{v}}\right|_{\max } \\
v_{U_{0}} \geq \frac{1}{2}(K+A)+\left|\Delta r_{\bar{v}}\right|_{\max }
\end{array}\right.
\end{aligned}
$$




\subsection{The Study of Zone Dividing Demodulator Boundary in Additive White Colored Noise Channel}

In communication, the influence of additive colored noise to the Hamilton modulated signals is more complicate than that of additive white noise, therefore the content is divided into two parts according to different processing to the colored noise: the first one is narrow-band additive gaussian white noise; the second one is all the other noise except the first one.

\subsubsection{The Solution to the Narrow-Band Additive Gaussian White Noise Channel}

As we know that the spectrum of white noise is a constant in the whole bandwidth, and the receiver of chaos M-ary communication system is a band-limited system because there is a band-limited fliter in the foreplane of radiofrequency. When the channel noise is ideal gaussian white noise, it will be changed into narrow-band gaussian white noise(it is not belong to independent and identically distributed noise, so it's narrow-band gaussian colored noise) after passing through the band-limited filter. In this situation, the problem of designing the boundary of zone dividing demodulator can be solved by selecting the proper sample points of the noise.

Due to the noise auto-correlation function is sinc function, if we sample the zero crossing interval of the sinc function, then all noise samples are uncorrelated and the joint density (likehood) function can be expressed as the product of independent terms. Therefore the designing method of zone dividing demodulator can be processed as the same in section 2.1.

\subsubsection{The Solution to the Other Colored Noise Channel except the Narrow-Band Additive Gaussian White Noise}

In this situation, it is different to study the noise's influence to the Hamilton modulated signals, in order to design the boundary of zone dividing demodulator. Therefore, we consider to change the colored noise into white noise by some ways, then the way of designing the zone divided demodulator in the additive white noise can be used to design the anti-colored-noise zone divided demodulator.

Generally the received signal from receiver can be expressed as follow

$$
r(t)=s_{c}(t)+n_{c}(t)
$$

where $s_{c}(t)$ denotes transmitted signal, $n_{c}(t)$ denotes additive colored noise. Althrough the colored noise can be whitened after $r(t)$ passed through the whitening filter, the signal will also be filtered, that is, the output signal is the prevailing input signal convolved with the impulse response of whitening filter. The boundary of zone dividing demodulator becomes hard to determine because the singal is interfered by the whitening filter. One solution is proposed here, which a pre-distortion filter is set up after the chaos baseband modulator in the transmitter and whitening filter is set up before zone dividing demodulator in the receiver, then the distortion signal after the whitening filter can be recovered and the colored noise can be whitened, the way of designing anti-white-noise zone dividing demodulator can be used in designing anti-colored-noise zone dividing demodulator.

Therefore, how to design whitening filter and pre-distortion filter has become the focal point in this research.

1) Whitening Filter

Without loss of generality, it is assumed that the stationary random process $X(t)$ has spectrum density $G_{x}(\omega)$, then $G_{x}(\omega)$ can be factored into its pole and zero constituents. 


$$
G_{x}(\omega)=a^{2} \frac{\left(\omega+Z_{1}\right) \ldots \ldots\left(\omega+Z_{n}\right)}{\left(\omega+\beta_{1}\right) \ldots \ldots\left(\omega+\beta_{m}\right)}, Z_{n} \neq \beta_{m}
$$

In the common engineering application, the spectrum density form of random signal is approximated to equation (20). Here $G_{x}(\omega)$ can be factored as

$$
G_{x}(\omega)=G_{x}^{+}(\omega)\left[G_{x}^{+}(\omega)\right]^{*}=G_{x}^{+}(\omega) G_{x}^{-}(\omega)
$$

where $G_{x}^{+}(\omega)$ contains all the poles and zeros that reside on the left side of S plane, $G_{x}^{-}(\omega)$ contains all the poles and zeros that reside on the right side of $\mathrm{S}$ plane. If there are zeros on the imaginary axis, these zeros must be in couple, then one zero can be put into $G_{x}^{+}(\omega)$, the othe zero will be put into $G_{x}^{-}(\omega)$.

The filter response can be obtained by using the factored power spectral density:

$$
\left|H_{1}(\omega)\right|^{2}=\frac{1}{G_{x}(\omega)}
$$

Due to

$$
\begin{gathered}
\left|H_{1}(\omega)\right|^{2}=H_{1}(\omega) H_{1}^{*}(\omega)=H_{1}(\omega) H_{1}(-\omega) \\
G_{x}(\omega)=G_{x}^{+}(\omega) G_{x}^{-}(\omega)=G_{x}^{+}(\omega) G_{x}^{+}(-\omega)
\end{gathered}
$$

Then we can get

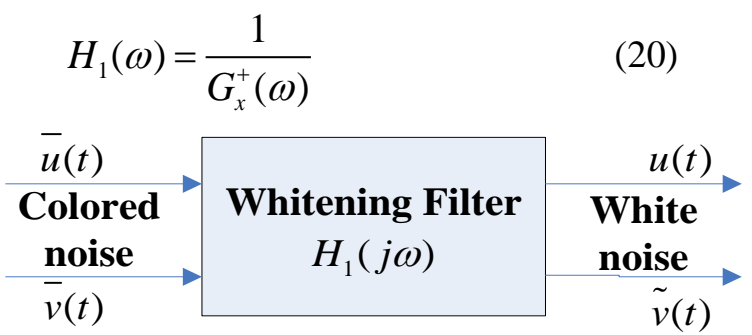

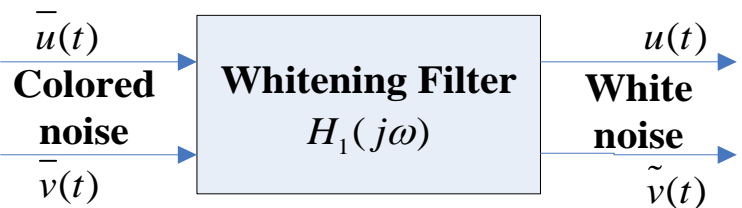

Figure 3. The Processing Structure of Whitening

As shown in Figure 3, the signal $\bar{u}(t)$ and $\bar{v}(t)$, which are filtered by the pre-distortion filter, are obtained by the radiofrequency demodulation and combined with colored noise. $u(t)$ and $\tilde{v}(t)$ are the recovered signal combined with white noise, then we can use the solution from section 2.1 to design the zone divided demodulator.

2) Pre-Distortion Filter

As we know that colored noise can be generated by processing stationary white noise with a system which has a transfer function with a finite number of poles and zeros, thus the pre-distortion filter response should be the inverse function of whitening filter.

\section{The Realization of Chaos M-ary Digital Communication System in Colored Noise Channel}

There are four main units on constructing chaos M-ary communication system in colored noise channel: Pre-distortion filter, whitening filter, zone dividing demodulator and symbol synchronization circuit.

\section{1) The Design of Pre-Distortion Filter}

As discussed in section 2, the pre-distortion filter response is the inverse function of whitening filter response, we assume that whitening filter response(stable filter) is: 


$$
H_{1}(z)=\frac{1}{G_{X}^{+}(z)}=\frac{A(z)}{B(z)}
$$

Then the pre-distortion filter response is

$$
H(z)=H_{1}^{-1}(z)=G_{X}^{+}(z)=\frac{B(z)}{A(z)}
$$

It is noticed that $H(z)$ is physically realizable filter(that is all poles and zeros must lie within the unit circle) and has inverse transformation, so the filter do not contain zero frequency. The Hamilton modulated signal which has zero frequency will be distorted when passing through this filter, therefore it is necessary to add up-converter unit for the Hamilton modulated signal, to ensure the modulated signal can be filtered without loss of frequency components.

\section{2) The Design of Whitening Filter}

The whitening filter should be physically realizable filter, and it can be set between radiofrequency demodulation and down-convert unit in the receiver, Its response can be expressed as:

$$
H_{1}(z)=\frac{1}{G_{X}^{+}(z)}=\frac{A(z)}{B(z)}
$$

\section{3) The Design of Zone Dividing Demodulator}

Although the demodulating principle are the same between each other, there are some differences between anti-colored-noise chaos communication and anti-white-noise chaos communication. Therefore it is necessary to get the formula form of zone dividing demodulator boundary.

After the process of deriving, the roughness of Hamilton modulated signal is expressed as:

$$
\left\{\begin{array}{l}
\left|\Delta r_{\bar{u}}\right| \leq\left|\Delta r_{\bar{u}}\right|_{\max }=\frac{1}{4} \beta \delta_{1} \sqrt{\frac{P_{s a v}}{\rho}}=0.35125 \alpha \beta \sqrt{\frac{J^{2}+K^{2}+A^{2}}{\beta \rho}} \\
\left|\Delta r_{\bar{v}}\right| \leq\left|\Delta r_{\bar{v}}\right|_{\max }=\frac{1}{4} \beta \delta_{2} \sqrt{\frac{P_{s a v}}{\rho}}=0.35125 \alpha \beta \sqrt{\frac{J^{2}+K^{2}+A^{2}}{\beta \rho}}
\end{array}\right.
$$

where $\left|\Delta r_{\bar{u}}\right|$ and $\left|\Delta r_{\bar{v}}\right|$ denote the roughness of Hamilton modulated signal in horizontal and vertical direction respectively, $J, K$ and $A$ are the paremeters of Hamilton modulated signal, $\rho$ denotes the the signal-to-noise ratio, $\alpha$ and $\beta$ denote the gain of up-convert and down-convert respectively.

Then the boundaries of zone dividing demodulator can be expressed as:

$$
\begin{gathered}
\left\{\begin{array}{l}
u_{L_{0}}=(\alpha \beta / 8) \times(J-A)-\left|\Delta r_{\bar{u}}\right|_{\max } \\
u_{R_{0}}=(\alpha \beta / 8) \times(J+A)+\left|\Delta r_{\bar{u}}\right|_{\max }
\end{array}\right. \\
\left\{\begin{array}{l}
v_{D_{0}}=(\alpha \beta / 8) \times(K-A)-\left|\Delta r_{\bar{v}}\right|_{\max } \\
v_{U_{0}}=(\alpha \beta / 8) \times(K+A)+\left|\Delta r_{\bar{v}}\right|_{\text {max }}
\end{array}\right.
\end{gathered}
$$


where $u_{L_{0}}$ and $u_{R_{0}}$ denote the left and right boundary of zone dividing demodulator, $v_{D_{0}}$ and $v_{U_{0}}$ denote the up and down boundary of zone dividing demodulator. The demodulator is set between down-convert and sample-hold unit in the receiver.

\section{4) The Design of Symbol Synchronization Circuit}

Due to the effect of channel noise, fading and multipath propagation, the output signal's waveform of baseband chaotic demodulator will arise jitters, peak or being sunken. In order to retrieve original digital information exactly, symbol synchronization clock shall be accurately extracted, then locates it to the middle position of every symbol period for extracting so that symbol jitters can be eliminated. Now the design of symbol synchronization circuit is given as shown in Figure 4.

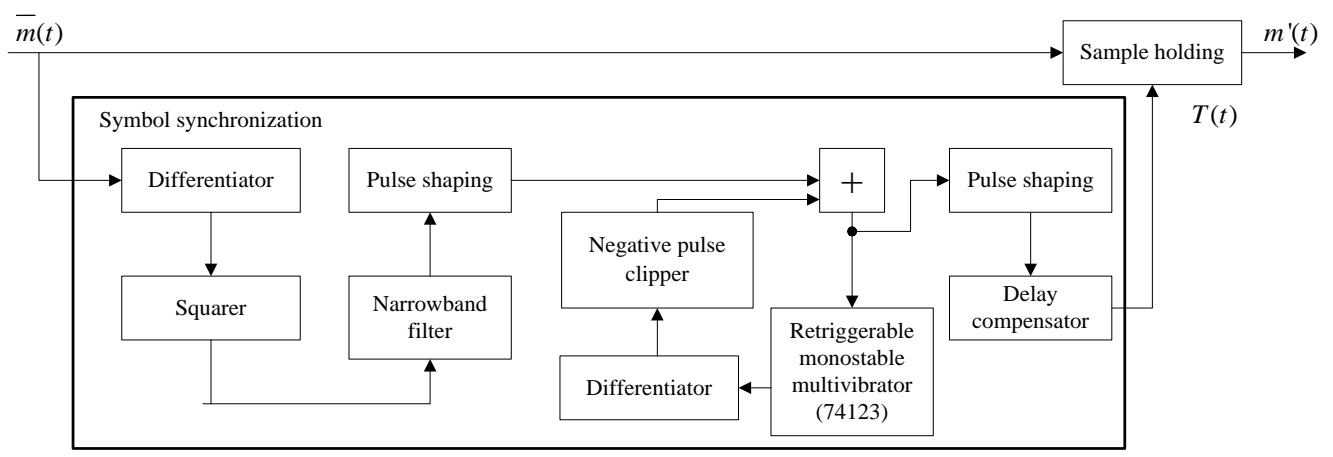

\section{Figure 3. The Design of M-Ary Chaotic Communication System Synchronization Circuit}

This synchronization circuit is on the basis of nonlinear transformation-filtering method, synchronization clock is extracted from the edge jumping information of symbol switching.

As shown in Figure 4, firstly differentiator is used to extract symbol edge information of baseband chaotic demodulation signal $\bar{m}(t)$. Next squarer is used to generate frequency multiplication components of symbol frequency, and narrowband filter is used for canceling channel noise and extracting frequency multiplication components of symbol frequency. After pulse shaping, symbol clock oscillates on the frequency multiplication components of symbol rate by synchronizing local oscillator. The local oscillator is a synchronized multivibrator composed of retriggerable monostable multivibrator, differentiator, negative pulse clipper and adder device, and it is mainly used for generating symbol synchronization clock automatically. The delay compensator aligns synchronization clock with center point of the received signal symbol period, to ensure symbol information $m^{\prime}(t)$ can be accurately retrieved by symbol extraction.

\section{Performance Simulation and Evaluation}

\subsection{The Performance Evaluation of Hamilton M-ary Chaotic Communication System in colored noise channel}

In order to evaluate the performance of anti-color-noise chaos $\mathrm{M}$-ary communication system, the Hamilton 4-ary chaotic communication system is constructed by employing the dynamic system analysis tool System View [13]. As shown in Figure 5, the P-D filter denotes the pre-distortion filter. 


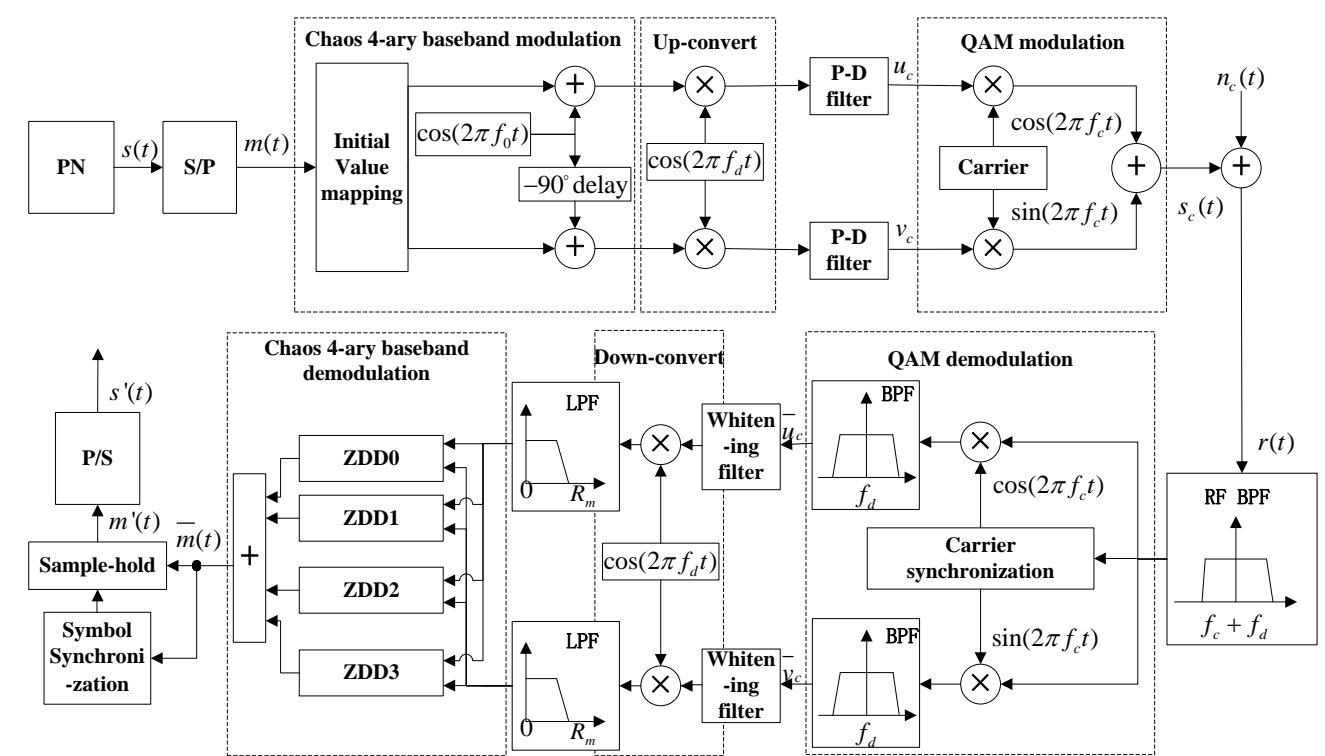

Figure 5. The Structure of Hamilton 4-Ary Anti-Color-Noise Chaotic Communication

The parameters are set as follows:

(1) The data source $s(t)$ is produced by a pseudo-random number (PN) sequence with rate $3.2 \mathrm{Mbps} . m(t)$ is 4 -ary signal after serial parallel conversion, whose symbol rate $R_{m}=1.6 \mathrm{Msps}$, i.e. one-symbol-interval $T=0.65 \mu \mathrm{s}$. Hamilton oscillator phase trajectory central point coordinates are $(4,-4),(4,4),(-4,4)$, $(-4,-4)$, corresponding to digital symbol information $0,1,2,3$, respectively. The amplitude and frequency of quadrature sine wave which is generating Hamilton oscillator modulation signal is $A=0.5 \mathrm{~V}$ and $f_{0}=1.6 \mathrm{MHz}$, respectively, upconvert frequency $f_{d}=20 \mathrm{MHz}$. The RF unit chooses QAM modulation, with frequency $f_{c}=50 \mathrm{MHz}$ and the amplitude is $1 \mathrm{~V}$.

(2) The channel chooses ACGN with power spectral density $N_{0}$.

(3) The central frequency and bandwidth of RF band-pass filter are $f_{c}+f_{d}=70 \mathrm{MHz}$ and $3.2 \mathrm{MHz}$ respectively. The central frequency and bandwidth of QAM demodulator filter which will recover the signal $\bar{u}(t)$ and $\bar{v}(t)$ are $f_{d}=20 \mathrm{MHz}$ and $3.2 \mathrm{MHz}$ respectively. The down-convert frequency is $f_{d}=20 \mathrm{MHz}$. The cut-off frequency of low-pass filter is $1.6 \mathrm{MHz}$. In order to compare with chaos $\mathrm{M}$-ary communication in white noise channel, the gain of upconvert and down-convert $\alpha$ and $\beta$ are all $2 \mathrm{~V}$.

(4) From equation (27),(28),(29) in section 4 it is known that the max roughness of Hamilton modulated signal $\left|\Delta r_{u}\right|_{\max }$ and $\left|\Delta r_{v}\right|_{\max }$ are all equal to $5.6419 \mathrm{~V}$, when SNR is 0 . It is bigger than the distance between each two signals' center which is equal to 4 . the phase trajectory of four signals will disturb with each other. However, when SNR increases, the roughness of signals' phase trajectory after QAM demodulation is decreasing. Therefore the boundary of zone dividing demodulator can choose the middle distance of two signals' phase trajectories: when $\overline{u_{t}(t)} \geq 0$ and $\overline{v_{t}(t)} \leq 0$, then the ZDD outputs 0 ; when $\overline{u_{t}(t)} \geq 0$ and $\overline{v_{t}(t)} \geq 0$, then the ZDD 
outputs 1 ; when $\overline{u_{t}(t)} \leq 0$ and $\overline{v_{t}(t)} \geq 0$, then the ZDD outputs 2 ; when $\overline{u_{t}(t)} \leq 0$ and $\overline{v_{t}(t)} \leq 0$, then the ZDD outputs 3 .

(5) The total system sample rate is $f_{S}=400 \mathrm{MHz}$. In $S N R=10 \mathrm{~dB}$, relative simulated waveforms obtained by SystemView are shown in Figure 6:

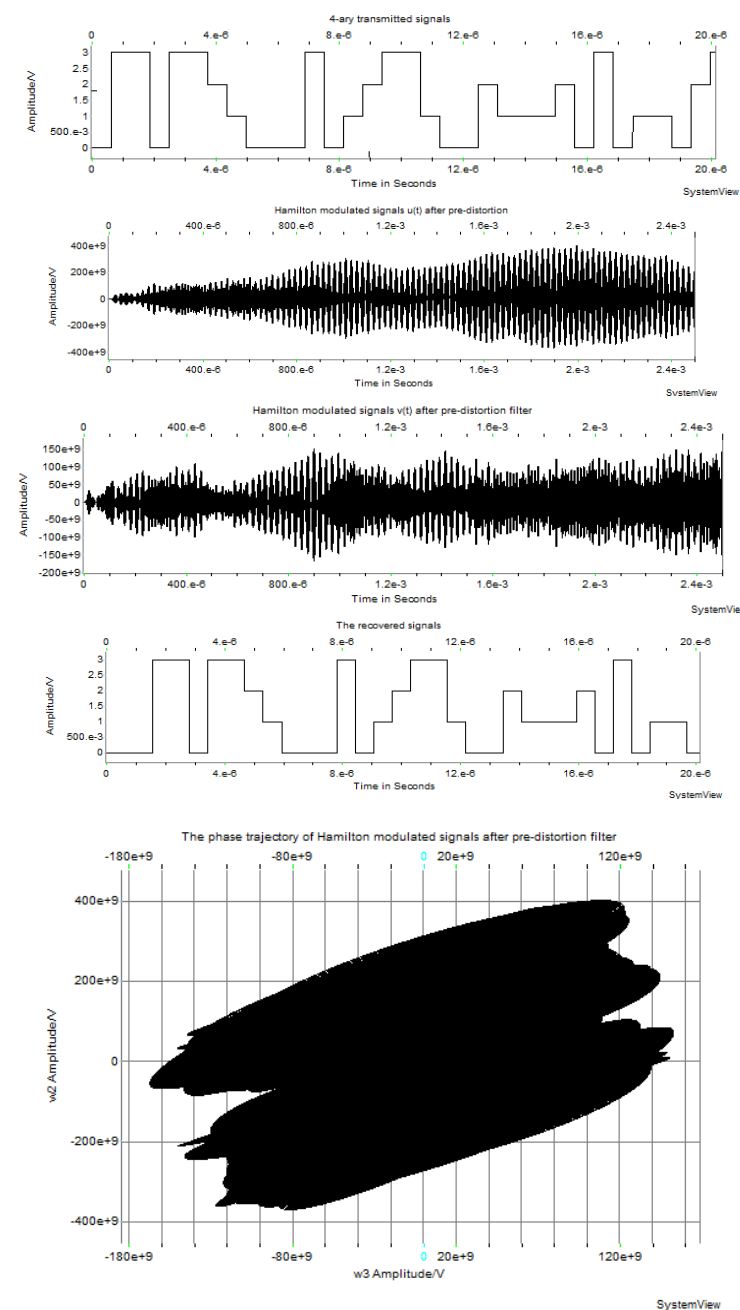

Figure 6. (A) 4-Ary Digital Information (B) Hamilton Modulated Signals U(T) After Pre-Distortion (C) Hamilton Modulated Signals V(T) After PreDistortion Filter (D) The Recovered 4-Ary Signals (E) The Phase Trajectory Of Hamilton Modulated Signals U(T) And V(T)

The first two error symbols in Figure 6(d) are caused by simulation system resolving delay, therefore they are not reckoned in symbol error rate statistics. The colored noise selected in this simulation is similar to blue and purple noise, whose gain is monotone increasing with the frequency. The initial gain of pre-distortion filter is $110 \mathrm{~dB}$, so the amplitude of the filter signals is large. As shown in Figure 6, the transmitted signals have been completely and exactly recovered by the ZDD in the colored noise channel, the modulated chaos signals $u(t)$ and $v(t)$ filtered by the pre-distortion filter are disorderly, aperiodic, the phase trajectories of chaos signal are also disorganized. Therefore the chaos signals have high security, and the validity of the constructed Hamilton chaos M-ary communication system in colored noise channel is verified. The proposed method of designing ZDD in colored noise is correct. 


\subsection{The Simulation Analysis of Symbol Error Characteristics of Hamilton M-ary Chaotic Communication System in Colored Noise Channel}

In order to evaluate the symbol error rate performance of the proposed Hamilton M-ary chaotic communication system in colored noise channel, the symbol average energy must be considered first. According to simulation parameter set up, the instant QAM carrier signal is expressed as follows:

$$
\begin{gathered}
s_{c}(t)=\left[u(t) \cos \omega_{c} t+v(t) \sin \omega_{c} t\right] \alpha \cos \omega_{d} t=\sqrt{u^{2}(t)+v^{2}(t)} \cos \left(\omega_{c} t-\theta_{m}\right) \alpha \cos \omega_{d} t \quad \text { (27) } \\
\text { where } \quad \theta_{m}=\arctan (v / u) \quad, \quad \omega_{d}=2 \pi f_{d} \quad, \quad \omega_{c}=2 \pi f_{c}, \\
f_{d}=20 \mathrm{MHz}, f_{c}=50 \mathrm{MHz}, \alpha=2 ; u(t)= \pm 4+0.5 \cos \Omega t, v(t)= \pm 4+0.5 \sin \Omega t, \text { in }
\end{gathered}
$$
which $\Omega=2 \pi f_{0}, f_{0}=1.6 \mathrm{MHz}$.

Thus, QAM symbol average transmitting power is:

$$
\begin{gathered}
P_{s a v}=\frac{1}{T} \int_{0}^{T} s_{c}^{2}(t) d t=\frac{1}{T} \alpha^{2} \int_{0}^{T}\left[u^{2}(t)+v^{2}(t)\right] \cos ^{2}\left(\omega_{c} t-\theta_{m}\right) \cos ^{2} \omega_{d} t d t \\
=\frac{1}{T} \int_{0}^{T}\left(16 \pm 4 \cos \Omega t+0.25 \cos ^{2} \Omega t+16 \pm 4 \sin \Omega t+0.25 \sin ^{2} \Omega t\right) \cos ^{2}\left(\omega_{c} t-\theta_{m}\right) 4 \cos ^{2} \omega_{d} t d t \\
=\frac{1}{T} \int_{0}^{T}(32.25 \pm 4 \cos \Omega t \pm 4 \sin \Omega t) d t \\
=32.25 \mathrm{~W}
\end{gathered}
$$

Therefore, in Hamilton oscillator M-ary chaotic QAM communication system, the ratio of symbol average energy to noisy power spectral density is expressed as

$$
\frac{E_{s a v}}{N_{0}}=\frac{P_{s a v} T}{N_{0}}=\frac{P_{s a v}}{N_{0} B}
$$

where $B=1 / T=R_{m}$ is equivalent baseband data bandwidth, $T$ is symbol period, $R_{m}=1.6 \mathrm{MHz}$ is symbol rate. $N_{0}$ is noisy power spectral density in the case of input impedance being $1 \mathrm{Ohm}$. According to (29), when $E_{\text {sav }} / N_{0}$ is set with $0,1, \cdots, 15 \mathrm{~dB}$ respectively, the corresponding channel noise power spectral density $N_{0}$ is calculated and given in Table 1. In order to evaluate the anti-colored-noise performance of the improved system, the symbol error rate of the proposed 4-ary chaotic communication as well as the original system proposed in [12] in different colored noise channel is drawn in Figure 7.

Table 1. The Simulation Parameters

\begin{tabular}{|c|c|c|c|}
\hline$E_{s a v} / N_{0}(\mathrm{~dB})$ & $N_{0}(\mathrm{~W} / \mathrm{Hz})$ & $E_{s a v} / N_{0}(\mathrm{~dB})$ & $N_{0}(\mathrm{~W} / \mathrm{Hz})$ \\
\hline 0 & $10.0781 \mathrm{e}-6$ & 8 & $1.5973 \mathrm{e}-6$ \\
\hline 1 & $8.0053 \mathrm{e}-6$ & 9 & $1.2688 \mathrm{e}-6$ \\
\hline 2 & $6.3589 \mathrm{e}-6$ & 10 & $1.0078 \mathrm{e}-6$ \\
\hline 3 & $5.0510 \mathrm{e}-6$ & 11 & $0.8005 \mathrm{e}-6$ \\
\hline 4 & $4.0122 \mathrm{e}-6$ & 12 & $0.6359 \mathrm{e}-6$ \\
\hline 5 & $3.4903 \mathrm{e}-6$ & 13 & $0.5051 \mathrm{e}-6$ \\
\hline 6 & $2.5315 \mathrm{e}-6$ & 14 & $0.4012 \mathrm{e}-6$ \\
\hline 7 & $2.0109 \mathrm{e}-6$ & 15 & $0.3490 \mathrm{e}-6$ \\
\hline
\end{tabular}




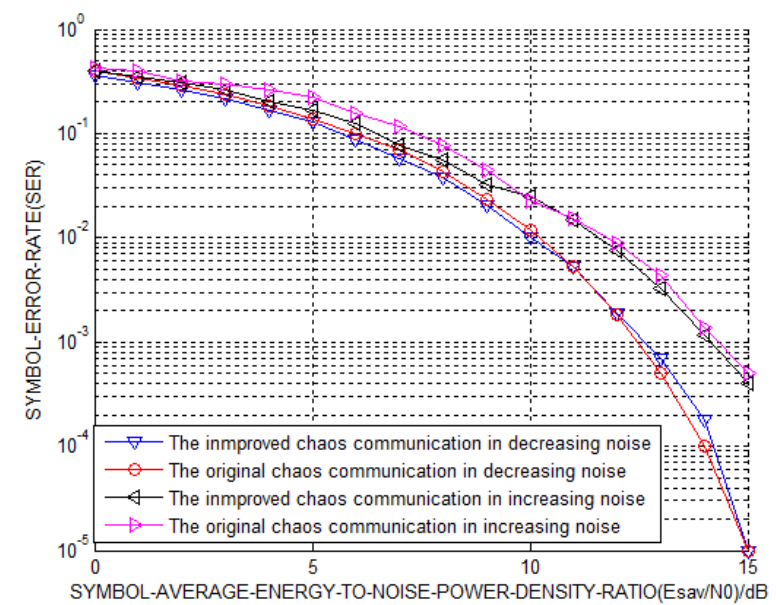

\section{Figure 7. SER Curves of Improved Hamilton Chaotic Communication System versus Original System in Different Colored Noise Channel}

In Figure 7, the improved chaos communication system is the anti-colored-noise system constructed, the original system is the system constructed in [12]. The increasing noise denotes the gain of the noise increases monotonically with frequency such as blue noise and purple noise; the decreasing noise denotes the gain of the noise decreases monotonically with frequency such as pink noise and brown noise. In decreasing noise channel, the symbol error rates of the two systems are similar to each other, when Esav/N0 is less than $10 \mathrm{~dB}$, the improved system's symbol error rate performance improves about $0.5 \mathrm{~dB}$ than that of original system. In increasing noise channel, when Esav/N0 is less than $10 \mathrm{~dB}$, the improved system's symbol error rate performance improves about $1 \mathrm{~dB}$ than that of original system, when Esav/N0 is greater than $10 \mathrm{~dB}$, its symbol error rate performance is still better than that of original system. The SER curves shows that the Hamilton chaos M-ary communication system proposed in this paper has better anti-colored-noise performance and has more secrecy than the original system.

\section{Conclusion}

In this paper, the method of constructing chaos M-ary communication by using Hamilton oscillator in colored noise is studied, and the design of the whole communication system is given. Meanwhile, the design of zone dividing demodulator in both additive white noise and colored noise channel is discussed in detail. The mathematical analysis of roughness of chaos signal in additive white noise channel is given, pre-distortion filter and whitening filter are applied to solve the design problem of zone dividing demodulator in additive colored noise channel. The Hamilton chaos 4-ary communication system in colored noise channel is constructed by employing SystemView, the validity and correctness of the proposed method are verified by the simulation. The result shows that the Hamilton chaos M-ary communication has performance improvement in anti-colored-noise. The superiority of the proposed system is that: the chaos signal is easy to generated, the system complexity is low, there is no need for chaos synchronization, the system has some security compared with traditional communication, and it has anti-noise performance improved.

\section{Acknowledgements}

This work was supported by National Natural Science Foundations of China (Grant Nos. 61172038) and Central University Research Business Expenses Special Fund. 


\section{References}

[1] W.H. Zhu, "Image encryption using CAT mapping and chaos approach", International Journal of Signal Processing, Image Processing and Pattern Recognition, vol. 7, no. 3, (2014), pp. 331-340.

[2] J.P. Bailey and A.N. Beal, "High-frequency reverse-time chaos generation using digital chaotic maps", Electronics Letters, vol. 50, no. 23, (2014), pp. 1683-1685.

[3] M.L. Barakat, and A.S. Mansingka, "Hardware stream cipher with controllable chaos generator for colour image encryption", IET Imag. Proceeding, vol. 8, no. 1, (2014), pp. 33-43.

[4] Q. Ye and L.L. Liang, "The Analysis and Application on a Fractional-Order Chaotic System", TELKOMNIKA (Telecommunication Computing Electronics and Control), vol. 12, no. 1, (2014), pp. 23-32.

[5] L.M. Pecora and T.L. Carroll, "Synchronization in chaotic systems", Physical review letters, vol. 64, no. 8, (1990), pp. 821-824.

[6] K.Y. Cheong, F.C. M. Lau and K.T. Chi, "Permutation-based M-ary chaotic-sequence spread-spectrum communication systems", Circuits, Systems and Signal Processing, vol. 22, no. 6, (2003), pp. 567-577.

[7] H. Wang, J. Guo and Z. Wang, "Chaotic M-ary direct sequence spread spectrum signals blind dispreading”, Journal of Tsinghua University, vol. 49, no. 1, (2009), pp. 13-16.

[8] M. Xu and H. Leung, "A Novel High Data Rate Modulation Scheme Based on Chaotic Signal Separation", IEEE Transactions on communications, vol. 58, no. 10, (2010), pp. 2855-2860.

[9] S. Zhu, Y. Xu and K. Yin, "Design of a Quadrature Differential Chaotic Phase Shift Keying Communication System", NSWCTC'09, International Conference on Networks Security, Wireless Communications and Trusted Computing, (2009), pp. 518-521, Wuhan, China.

[10] T.J. Wren and T.C. Yang, "Orthogonal chaotic vector shift keying in digital communications", IET communications, vol. 4, no. 6, (2010), pp. 739-753.

[11] S. Arai, Y. Nishio and T. Yamazato, "M-ary modulation scheme using separation of chaotic dynamics for noncoherent chaos-based communications", Proc. NOLTA'09, (2009), pp.312-315.

[12] Y.Q. Fu and X.Y. Li, "Chaos M-ary modulation and demodulation method based on Hamilton oscillator and its application in communication", Chaos: An Interdisciplinary Journal of Nonlinear Science, vol. 23, no. 1, (2013), 013111.

[13] SystemView (Advanced Dynamic System Analysis) User Manual, California USA: ELANIX Company, (1999), pp. 1-291. 